complexity. With time, Benzer himself realized that Drosophila are not behavioural atoms but rather intricate individuals. $\mathrm{He}$ abandoned behavioural for developmental analysis.

No doubt, Weiner was captivated by the eccentric style of Benzerism, and this encouraged him to recite scores of amusing anecdotes. Whether all appeal to the general public, or only to a small cult, is a different question.

Another caveat is also appropriate: students who read the book should not expect mainstream science to follow Benzer's model. Most laboratory work is much more boring than the hunt for bizarre mutants in the forefront of science. Most mentors are much less imaginative, daring and permissive than Benzer. And, unfortunately, the days are long gone when an enthusiastic investigator shared plans without fear, and mutants without a lawyer. This was before business killed fair play.

As for Benzer himself - at the age of 77 he has just discovered a new mutant, Methuselah, that refuses to age.

Yadin Dudai is at the Weizmann Institute of Science, Rehovot 76100, Israel.

\section{Hitting the target}

\section{The Elusive Magic Bullet}

by John Mann

Oxford University Press: 1999. 202 pp.

$\mathfrak{E} 18.99, \$ 29.95$

\section{Stephen Neidle}

The use of medicines to combat disease almost certainly predates recorded human history. One can imagine our prehistoric ancestors, during the course of foraging for food, finding that whereas some plants and fruits were harmful, others could have beneficial effects. The Chinese developed particular expertise with herbal remedies, a tradition that is at least 5,000 years old and still thrives today.

The science of drug discovery has its origins in this tradition. Many subsequent cultures also contributed, with primitive pharmacy arising from the ancient Greeks, and notably from the Arabic world. The Dark Ages onwards saw these elements intertwining with the pseudo-science of alchemy, a combination that dominated materia medica until the onset of modern science at the end of the eighteenth century. All this and more was the subject of John Mann's earlier book, Murder, Magic and Medicine, which focused on the use of natural products through the ages, for both base and noble purposes. Mann, a distinguished natural product chemist himself, succeeded in the difficult task of writing an entertaining yet

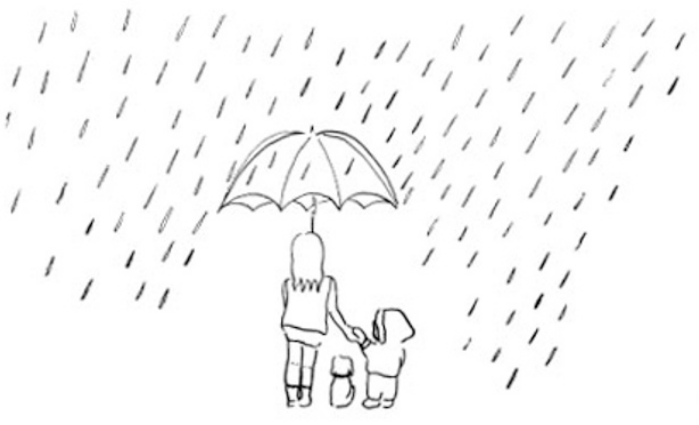

\title{
Tracing the development of lines
}

The artistic development of talented and less talented children was compared over a ten-year period by the developmental psychologist Constance Milbrath. She assessed the ages of development of different elements of drawing, including form, spatial relationships and composition. The study has been written up in a technical book, Patterns of Artistic

erudite book, with appeal to readers across a wide spectrum of backgrounds.

His new book continues and expands on this theme and approach. Its focus is the discovery of twentieth-century medicines for the treatment of bacterial and viral diseases and cancer, and their historical context. Its style is a beguiling blend of science and historical detail, liberally spiced with anecdotes and accounts of the wider impact of these discoveries.

The "magic bullet" concept originated with Paul Ehrlich. Mann pays fitting tribute to Ehrlich as the true father of modern approaches to drug discovery, since he also effectively devised the concepts of therapeutic index, chemoreceptors and combination therapy. But it is the idea that there exist perfectly specific drugs to combat human disease that grabs the public imagination. One real strength of this book is Mann's critical examination of this idea, especially in the context of anti-cancer therapies, where public demand for magic bullets is a potent force.

The survey of biological approaches to cancer therapy succinctly covers such topics as interleukins, Ras farnesyltransferase inhibitors, angiogenesis and antibodydirected enzyme prodrug therapy. As Mann points out, this last approach probably comes closest to Ehrlich's original concept, even though it still awaits full verification in the clinic.

It is difficult to envisage a world without

Æ๑) 1999 Macmillan Magazines Ltd
Development in Children: Comparative Studies of Talent (Cambridge University

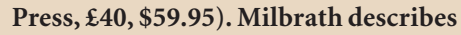
the development of artistic talent as being dependent on both figurative and cognitive abilities. Here, a talented 11-year-old has drawn the back views of two people and a dog.

even the simplest antibacterial agents to ensure that we don't succumb to a brief encounter with pathogens in our everyday life. Average life expectancy was only about 45 years at the turn of this century, when Alexander Fleming started as a medical student. One child in six did not survive beyond five years. Mann has provided a vivid account of the devastating impact that bacterial diseases had on the populace, right up to the Second World War, and the dramatic effects of the widespread availability of antibiotics such as penicillin and streptomycin. Some topics, such as the discovery of penicillin and its subsequent development into a clinically available drug, are rightly given extensive coverage. The problems of drug resistance and superbugs are also discussed in brief, but adequate, illustrated descriptions of antibiotics' modes of action and the molecular basis of resistance.

Surprisingly, in a book written by a chemist, there is not a single chemical structure to be found, even though there are numerous descriptions of them and their significant features. Perhaps the publishers felt that the inclusion of chemical formulae would intimidate potential purchasers.

Following the Second World War, the search for further chemotherapeutic agents with selective activity against particular organisms became the mainstay of much of the pharmaceutical industry. Many companies were effectively vast palaces of organic 
chemistry and microbiology, to which other biological sciences were mere appendages providing the necessary in vitro and in vivo screens. Studies of the products of mould and microorganism metabolism and their chemistry dominated drug research. This approach resulted in the discovery of many effective antibacterial and some useful antitumour agents. Others often resulted from chance findings, notably the anticancer drug cisplatin.

However, by the late 1980s drug discovery was in a rut, with the realization that random screening was an expensive and ultimately unscientific way to find agents with significantly superior activity and selectivity. The subsequent revolution in our understanding of the molecular basis of many diseases has prompted fundamental changes in approach. The current, more rational approach is based on an understanding of pathogen and malignant cell and molecular biology. The impact of the human genome project on the treatment of human disease promises to be even more profound.

Mann's parting message is that all this takes us full circle, back to the essence of Ehrlich's approach, based on the idea of specific chemoreceptors.

Inevitably, a small book with such an ambitious agenda has to emphasize some topics more than others. I would have expected to see a fuller (and illustrated) account of the impact of structure-based drug design, especially since there are several excellent recent examples now in the clinic, such as the anti-HIV protease inhibitors. There is no real mention of combinatorial chemistry, now having a profound impact in industry and even academia.

However, these are minor caveats for an engrossing book which serves its intended audience well. It has much of value for the interested layperson, as well as students and even professionals wishing to have a gentle yet erudite introduction to the past, present and future of chemotherapy.

Stephen Neidle is in the CRC Biomolecular Structure Unit, The Institute of Cancer Research, Sutton, Surrey SM2 5NG, UK.

\section{Drugs from other perspectives On the one hand:}

Bitter Pills: Inside the Hazardous World of Legal Drugs

by Stephen Fried

Bantam Books, $\$ 24.95$

And on the other...

In Quest of Tomorrow's Medicine: An Eminent Scientist Talks About the

Pharmaceutical Industry, Biotechnology, and the Future of Drug Research [Die verspielte Zunkunft]

by Jürgen Drews, translated from the German by David Kramer

Springer, $\$ 29.95, £ 22.50$

\section{Taming the black dog}

\section{Malignant Sadness: The Anatomy of Depression}

by Lewis Wolpert

Faber \& Faber: 1999. 186 pp. £9.99

\section{Charles B.Nemeroff}

In the past decade, a number of books have documented personal experiences with depression or manic-depressive illness. There are the rare public figures willing and able to share their voyages to hell and back. There are the professionals (including mental-health workers) who have, after an episode of depression, dramatically changed their view of the disorder. And there are the articulate patients wishing to educate both the lay public and health-care professionals on the diagnosis and treatment of this devastating disease. There are probably more than 20 such volumes available, many of them excellent. Do we need another? In contemplating the request to review this book, I had to take into consideration my current tardiness in several projects. So why did I agree?

For the biologists, the answer is obvious, but to the rest of you, it is simply because the author is Lewis Wolpert. Wolpert is an accomplished developmental biologist, who has not only made remarkable research contributions, but has helped frame many questions in biological research, cutting across a number of disciplines. In view of his scientific prowess and his role as a philosopher- scientist, it was simply too tantalizing for me to resist the temptation to preview what he writes about a disorder that I have spent most of my professional life studying.

I was not disappointed; there is much good here. As he himself now recognizes, one of the major problems with depression is the stigma attached to it by both the public and many health-care providers. His willingness to share the pain, self-doubt, hopelessness and helplessness that is pathognomonic of this disorder will help many non-sufferers of depression to understand, and many depressed patients to fight this "black dog", as Churchill termed it. Wolpert's description of the severe sleep disturbance, obsession with suicide, anxiety, inability to concentrate, diurnal mood variation and bewildering physical symptoms is compelling. His grasp of the literature is, not surprisingly considering his biological background, very topical and generally accurate. He conveys the public-health implications of depression in a very readable manner, which is no small task. He highlights several facets of depression that are generally not emphasized enough, including its remarkably high prevalence worldwide, and the morbidity and mortality associated with it. In the United States, suicide is the ninth leading cause of death, in spite of the experts' acknowledgement that many, if not most, suicides go unreported.

Wolpert's repeated emphasis on depression as a systemic disease that affects several other organs, including the heart, is a wellestablished finding that has not received the recognition it deserves. He is one of the first authors to repeatedly remind us that the

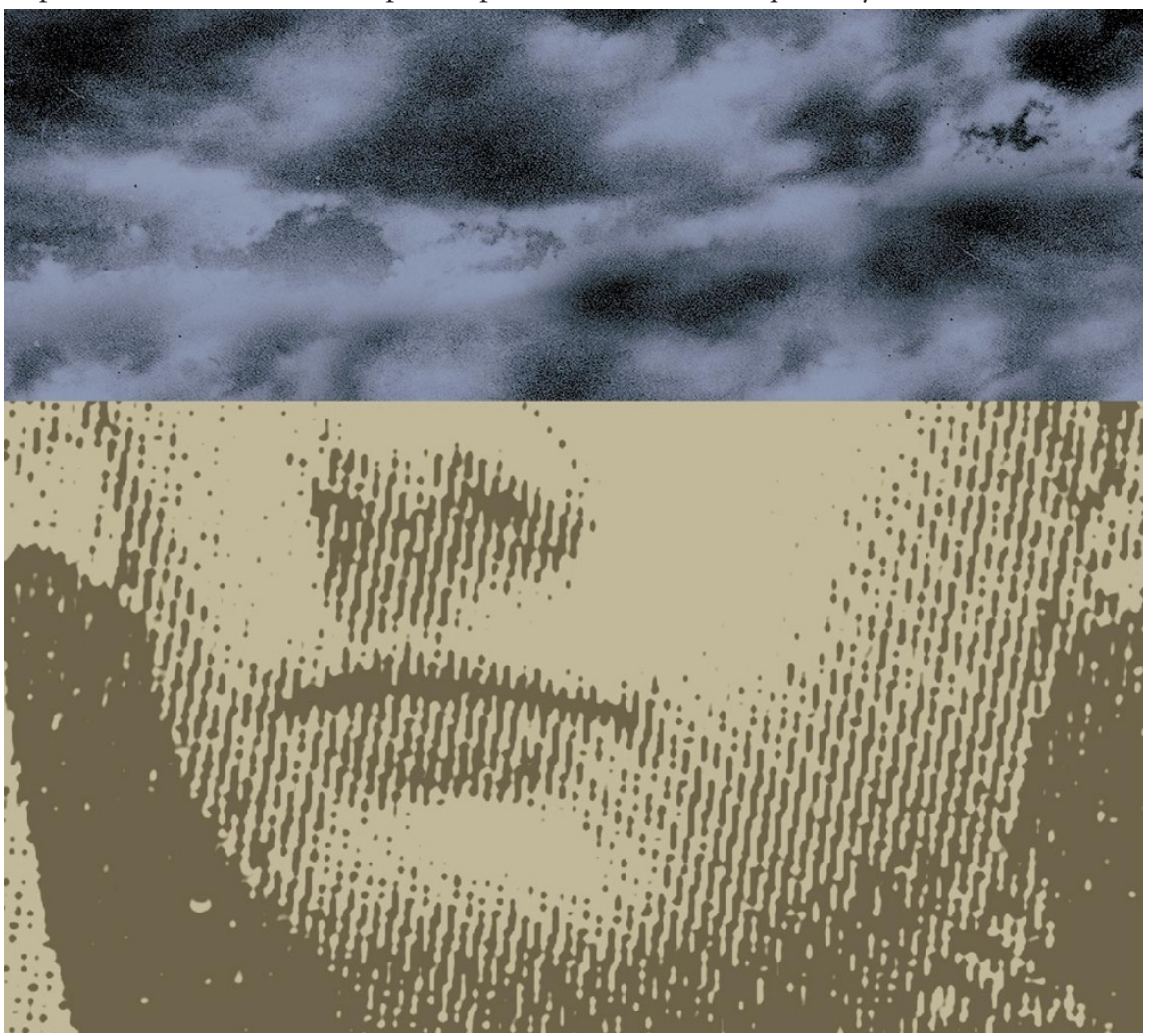

